\title{
The 5G Localization Waveform Ranging Accuracy over Time-Dispersive Channels - An Evaluation
}

\author{
Emanuel Staudinger, Michael Walter, and Armin Dammann \\ German Aerospace Center (DLR) \\ Institute of Communications and Navigation \\ Oberpfaffenhofen, 82234 Wessling, Germany \\ Email: \{emanuel.staudinger\}@dlr.de
}

\begin{abstract}
Localization has increasingly become important for a variety of applications and context aware services. Today's mobile communication terminals exploit existing reference signal structures for propagation delay based positioning. Recently, particular single-parametrized waveforms with adaptable power spectral densities (PSDs) haven been proposed in the context of 5G. These waveforms haven been investigated based on CramérRao lower bounds (CRLBs) and Ziv-Zakai lower bounds (ZZLBs) for multipath-free channels.

Time-dispersive channels have neither been investigated theoretically nor numerically. In this work, we make this gap smaller by numerical evaluations of the proposed waveforms. We focus on a simple correlation-based receiver and investigate the resulting ranging error. Our evaluations with varying root mean square (RMS) delay spread and fixed Rician K-factor clearly show, for which particular channels and signal bandwidths specific waveforms and their respective parameters should be chosen. A ranging error reduction of factor 1.2 to more than 5 compared to state of the art reference signals can be obtained. Hence, we pave the way to possibly place a new waveform within the 5G context for improved ranging accuracy compared to state of the art.
\end{abstract}

\section{INTRODUCTION}

Position information has increasingly become important for a variety of applications and context aware services. Global navigation satellite systems (GNSSs) are commonly used to provide position information outdoors. The required localization accuracy and precision highly depend on the intended application. Only outdoors with little multipath, specialized GNSS receivers provide centimeter-accuracy and sufficiently high position information update rates required for automated machinery. Strong multipath, signal blockage, and unfavorable geometric satellite constellations result in highly degraded positioning accuracies. Alternative wireless systems are therefore used to complement, or even replace GNSS based positioning. State of the art communication standards, such as $3 \mathrm{G}$, and $4 \mathrm{G}$ already provide various positioning methods to complement GNSS based localization for mobile terminals. These methods infer position information based on ranging, connectivity, or angular information. Additionally, cooperative localization techniques utilizing device-to-device (D2D) connectivity have shown a superior positioning performance in dense networks [1]. However, localization is not considered in wireless communication standards targeting mass-market from the beginning of the design phase, as for example ranging is commonly seen as side-product within the communication community. The only exception up till now are the latest releases of the 3rd Generation Partnership Project (3GPP)-Long Term Evolution (LTE) for which specific signal structures have been squeezed into the standard, without considering specific optimized waveforms and localization requirements [2].

Recent developments within the wireless communication community to revolutionize the existing $4 \mathrm{G}$ communication standard towards $5 \mathrm{G}$ pave the way to actually place localization into an upcoming 5G standard from the beginning of the design phase [3]. Various optimized waveforms are currently discussed to solely improve communication in terms of throughput, latency, adjacent channel interference, and coverage [4]. These optimized waveforms mostly cover existing concepts based on filtered multi-carrier modulation, or filterbank based modulation, and would allow a flexible spectrum usage. A key focus within $5 \mathrm{G}$ is also on machine-to-machine communication and collaborative communication networks, which plays in our hand for cooperative positioning. Massively connected mobile terminals with D2D wireless links would become available, and the authors in [5] already showed the theoretical benefit of high mobile terminal densities for localization. However, all envisaged $5 \mathrm{G}$ waveforms so far do not consider any ranging aspect and assume a rectangular signal power spectral density (PSD).

Recently, a 5G localization waveform has been proposed to trade-off ranging precision for ranging signal miss-detection during initial acquisition. In [6] the authors evaluated the ranging precision of a set of single-parametrized waveforms with different PSDs based on Cramér-Rao lower bounds (CRLBs) and Ziv-Zakai lower bounds (ZZLBs). They focused on multipath-free channels with additive white Gaussian noise (AWGN) but without shadow fading. As a result they showed that particular waveforms can be chosen to improve ranging precision compared to a state of the art rectangular PSDs in signal-to-noise ratio (SNR) limited scenarios. This parametrized waveform concept has been extended in [7] to evaluate the positioning performance in a non-cooperative scenario with three base stations and one mobile station. Again, the channel has been assumed multipath-free and without shadowing, and a maximum achievable gain of $42.3 \%$ has been found. Further investigations included path loss and shadow fading from WINNER II channel models to obtain 
more realistic results. However, no multipath resulting in a frequency-selective channel is assumed.

In reality ranging accuracy is mostly degraded by multipath in combination with low to medium bandwidth-limited signals for terrestrial mobile communication systems, and not by SNR. Naturally, we focus on investigating the ranging accuracy of the newly proposed 5G localization waveform for frequencyselective channels in this work. We particularly chose a lowcomplex estimator estimating the first arriving path only. Hence, we will show how well suited the proposed waveform will be for ranging with bandwidth-limited signals, and which parameters should be chosen.

Our contribution is organized as follows: in Sec. II we recall the proposed parametrized waveforms, followed by the transmission model for time-of-arrival (ToA) based ranging in Sec. III. The derived maximum-likelihood (ML) estimator is presented in Sec. IV and we first investigate the multipath error envelope in Sec. V. In Sec. VI we apply different WINNER II channel models to evaluate the ranging accuracy, followed by a more generalized evaluation with varying root mean square (RMS) delay spreads in Sec. VII. Finally, we wrap up our work in the conclusion and give a possible outlook for further investigations in Sec. VIII.

\section{5G LOCALIZATION WAVEFORMS}

The authors in [6] proposed several single-parametrized ranging waveforms with different power spectral densitys (PSDs). In general, these waveform proposals can be viewed as windowing functions. For clarity, we shortly recall those waveforms as they provide the basis for our investigations.

The first parametric waveform consists of a triangular shaped PSD as depicted in Fig. 1(a). We can analytically describe the PSD with a so called shaping parameter $\alpha \in \mathbb{R}$, $0 \leq \alpha \leq 1$ as

$$
|S(f)|^{2}= \begin{cases}(1-\alpha) \frac{2}{B}-\frac{4(1-2 \alpha)}{B^{2}}|f|, & |f| \leq \frac{B}{2} \\ 0, & |f|>\frac{B}{2}\end{cases}
$$

with the signal bandwidth $B$ [6]. For an $\alpha=0.5$ we obtain a state of the art uniform PSD, which we refer to as rectangular waveform throughout this paper. Furthermore, this rectangular waveform serves as reference. The corresponding autocorrelation functions are derived in [6], and Fig. 1(b) shows the resulting autocorrelation functions for the waveforms depicted in Fig. 1(a). One aspect is directly visible from Fig. 1(b): we trade-off the width of the main correlation peak for the height of the decaying side-lobes similarly to using windowing functions in spectral estimation. Consequently, the power of interfering multipath components from a time-dispersive channel can either be suppressed or even increased, depending on the delay relative to the line-of-sight $(\mathrm{LoS})$ path.

As second parametrized waveform we consider a weighted superposition of two Dirac delta functions at the spectrum's edge and a rectangular waveform. This superposition has been beneficial in [6] to decrease the CRLB. We refer to

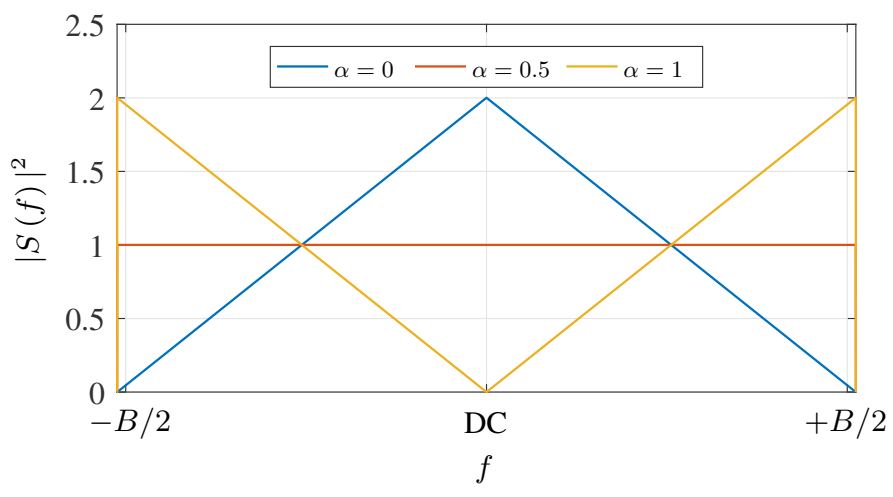

(a) Power spectral density $|S(f)|^{2}$ for different parameters $\alpha$.

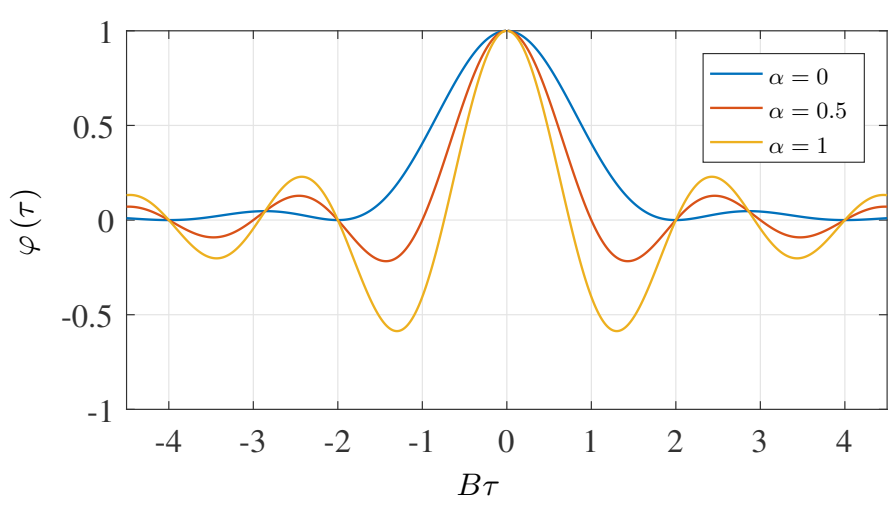

(b) Autocorrelation function $\varphi(\tau)$ for different parameters $\alpha$.

Fig. 1. Triangle waveform [6].

this waveform as Dirac-rectangular waveform and denote the power spectral density as

$$
|S(f)|^{2}= \begin{cases}\frac{1-\gamma}{B}+\frac{\gamma}{2}\left[\delta\left(f+\frac{B}{2}\right)+\delta\left(f-\frac{B}{2}\right)\right], & |f| \leq \frac{B}{2} \\ 0, & |f|>\frac{B}{2}\end{cases}
$$

with the signal bandwidth $B$, and $\gamma \in \mathbb{R}, 0 \leq \gamma \leq 1$ as shaping parameter [6]. Fig. 2(a) illustrates the PSD. A rectangular waveform is obtained by setting $\gamma=0$. Fig. 2(b) shows autocorrelation functions for different $\gamma$. Compared to the triangular waveform, the side-lobes of the autocorrelation function do not decay. In the extreme case with $\gamma=1$ the resulting autocorrelation is a cosine-function. Hence, the usable parameter range will be different for time-dispersive channels compared to an AWGN channel without multipath, as evaluated in [6].

The two afore introduced waveforms showed, comparable to windowing functions used in spectral estimation, a trade-off between the width of the main correlation peak and the height of the side-lobes. It is hence desirable to use a waveform with controllable side-lobe amplitudes. Dolph-Chebyshev window functions fulfill this condition and represent the third evaluated waveform within this work. Fig. 3(a) and Fig. 3(b) illustrate the PSD and the autocorrelation function respectively. We denote a shaping parameter $\kappa \in \mathbb{R}, \kappa>0$ in Decibel to define 


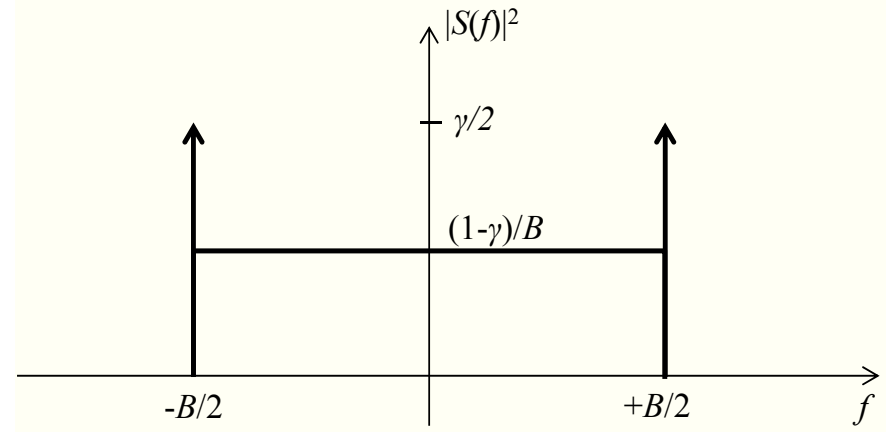

(a) Power spectral density $|S(f)|^{2}$ [6].

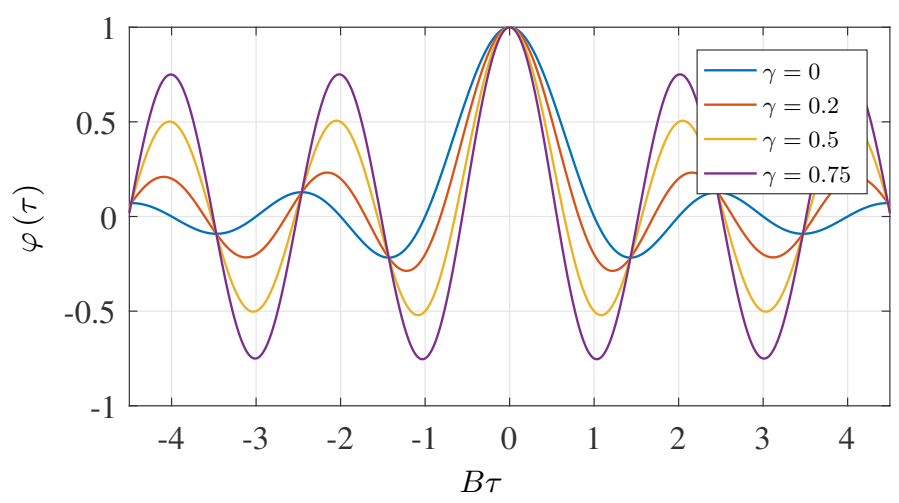

(b) Autocorrelation function $\varphi(\tau)$.

Fig. 2. Dirac-rectangular waveform.

the side-lobe attenuation. Further derivations and waveform definitions are available in [6].

At the end of this section we want to relate these waveforms to the latest developments and envisaged improvements in $5 \mathrm{G}$ : in $5 \mathrm{G}$ massively connected devices, either mobile terminals for persons or for machines, enable cooperative positioning techniques. System bandwidth and transmission power are very limited for carrier frequencies below $6 \mathrm{GHz}$. Hence, more optimal waveforms to improve ranging accuracy for cooperative positioning compared to state of the art would significantly improve mobile terminal localization. As stated in [6], only the PSD is of interest. Consequently, phases of subcarriers can either be modulated with data symbols or optimized for a reduced peak-to-average power ratio (PAPR). Within 5G various different modulation schemes are proposed to enable a flexible spectrum's usage and to enable more time- and frequency-concentrated signals, e.g., reducing adjacent channel interference. Single-parametrized waveforms for ranging can easily be placed within this context.

\section{TRANSMISSION MODEL}

Our transmission model depicted in Fig. 4 comprises a multi-carrier transmitter and a multi-carrier receiver based on orthogonal frequency division multiplex (OFDM). Transmitter and receiver are perfectly synchronized in time to enable ToA or time-of-flight (ToF) ranging. The PSD of the transmitted signal is denoted as $|S(f)|^{2}$ with $f \in \mathbb{R},-B / 2 \leq f \leq B / 2$.

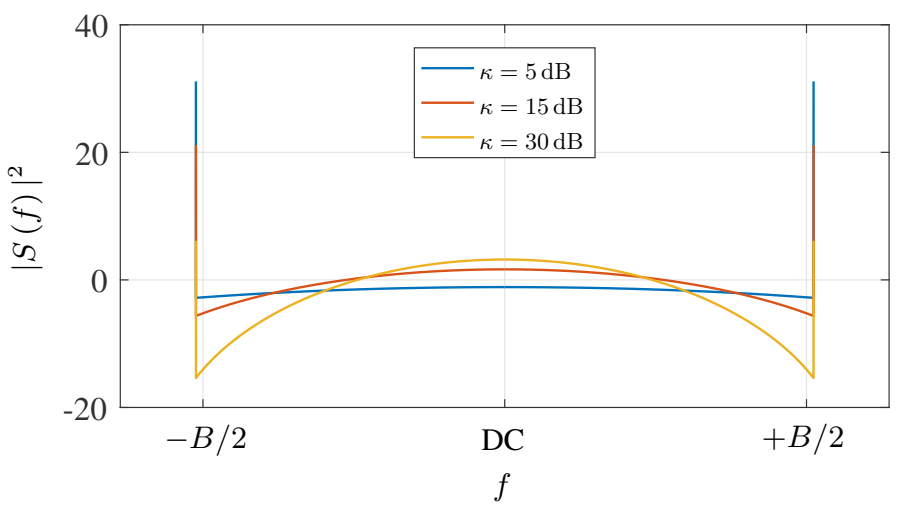

(a) Power spectral density $|S(f)|^{2}$ for different parameters $\kappa$.

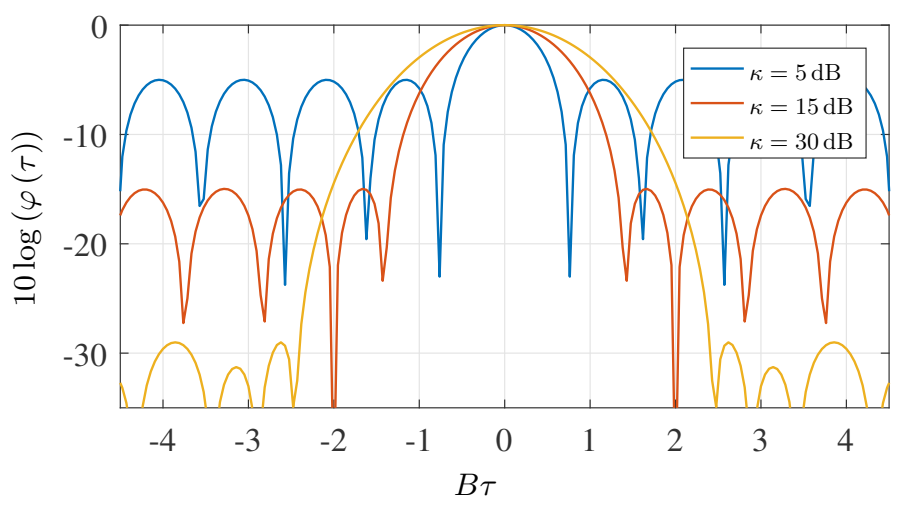

(b) Autocorrelation function $\varphi(\tau)$ for different parameters $\kappa$.

Fig. 3. Dolph-Chebyshev waveform.

Assuming a sampling interval $T_{\mathrm{s}}$ as the inverse of the sampling frequency $f_{\mathrm{s}}$, we can describe the sampled and transmitted OFDM symbol in time-domain as

$$
s[n]=\frac{1}{\sqrt{N}} \sum_{l=-N / 2}^{N / 2-1} S\left(l \frac{f}{f_{\mathrm{s}}}\right) \mathrm{e}^{\mathrm{j} 2 \pi n \frac{l}{N}},
$$

with $n$ as sample index in the range $n=[-C, N-1]$, and $l$ as subcarrier index. The OFDM symbol consists of $N$ even subcarriers and the cyclic prefix is $C$ samples long. In practical implementations we need to keep guard bands at the spectrum's edge for low-pass filtering. Hence, only $N_{\mathrm{u}}<N$ usable subcarriers can be allocated, but the power of the transmitted signal $s[n]$ is normalized to one. An in general time-dispersive mobile radio channel can be expressed as summation of $M$ weighted Dirac delta functions $\delta$

$$
h[n, \tau]=\sum_{m=1}^{M} \beta_{m} \delta_{m}\left[n-\frac{\tau_{m}}{T_{\mathrm{s}}}\right],
$$

with $\beta_{m} \in \mathbb{C}$ as complex amplitude for the multipath component $m$, and $\tau_{m} \in \mathbb{R}^{+}$as delay of multipath component $m$ in seconds. The LoS component is represented by $m=1$, and we assume a stationary channel for the duration of one OFDM ranging symbol. Gaussian distributed white noise with 


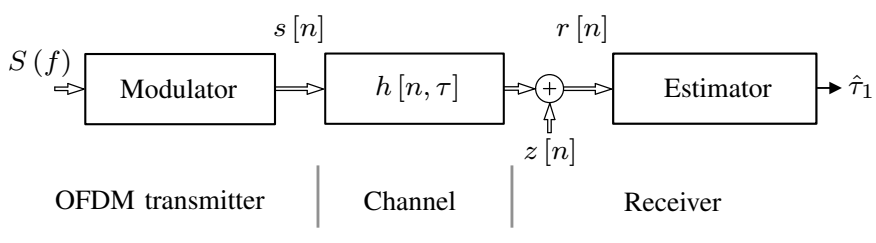

Fig. 4. Transmission model for OFDM based ToA ranging.

zero mean and variance $\sigma_{z}^{2}$ represented by $z[n]$ is added, and we obtain the received signal

$$
r[n]=s[n] * h[n, \tau]+z[n]
$$

at the receiver. The estimator derived in the next section is used to estimate the delay of the first incoming path $\tau_{m=1}$ denoted as $\hat{\tau}_{1}$.

\section{MAXIMUM-LiKELIHOOD ESTIMATOR}

The estimator in Fig. 4 consists of an maximum-likelihood (ML) estimator assuming a mobile radio channel with a singletap only. As shown in [8], this ML estimator can be realized as single channel-tap correlation receiver, equivalent to a delaylocked loop (DLL) used in GNSS receivers. We focus on snapshot based estimation, where only one received OFDM symbol is used for estimation. No tracking is applied. The ML estimator to incoherently estimate the delay of the LoS path therefore corresponds to

$$
\hat{\tau}_{1}=\underset{\tilde{\tau}_{m}}{\arg \max }\left|\sum_{l=0}^{N-1} r^{*}[l] s_{\text {ref }}\left[l-\frac{\tilde{\tau}_{m=1}}{T_{\mathrm{s}}}\right]\right|,
$$

assuming an unknown phase offset between transmitter and receiver. The correlation length is equivalent to the OFDM symbol length without the cyclic prefix. The received signal $r[n]$ has been defined in (5), and the time-delayed reference signal for crosscorrelation in the index range $l=[0, N-1]$ is defined as

$$
s_{\text {ref }}\left[l-\frac{\tilde{\tau}_{1}}{T_{\mathrm{s}}}\right]=\frac{1}{\sqrt{N}} \sum_{x=-N / 2}^{N / 2-1} S\left(l \frac{f}{f_{\mathrm{s}}}\right) \mathrm{e}^{\mathrm{j} 2 \pi n \frac{l-\tilde{\tau}_{1} / T_{\mathrm{s}}}{N}} .
$$

Range estimation is commonly divided into two steps: an acquisition step and a fine-synchronization step. In the acquisition step the correlation function in (6) is evaluated on a time grid which is integer multiples of the receiver's sampling duration $T_{\mathrm{s}}$. The maximum of this coarse-grid correlation function is detected and used as initialization for fine-synchronization. By using OFDM we can shift the finesynchronization from time-domain into frequency-domain to avoid costly signal interpolations in time-domain for subsample delay estimation. The cyclic prefix enables a shift from linear to circular convolution. Hence, we can estimate the delay in frequency-domain. Ranging performance therefore depends on both steps. Correlation functions with high sidelobes result in incorrect maximum correlation peak detection during the acquisition phase, and ranging estimates become erroneous.
We can see from (6) that we optimize with respect to the strongest path only, assuming that the LoS path used for ranging is the strongest path. The channel itself as defined in (4) comprises $M$ components. As a result, our ML estimator will be biased and the ranging performance of the ML estimator cannot be assessed with lower bounds such as the CRLB or the ZZLB anymore. Consequently, we are interested how the estimation bias changes with respect to the signal's PSDs defined in Sec. II if we keep this simple estimator. Is it possible to obtain a lower estimation bias with waveforms different from the rectangular waveform? Throughout the rest of this paper we refer to estimation accuracy as the bias, and to precision as the variance/standard deviation.

\section{Multipath Error EnVelope}

In general, time-dispersive wireless channels affect any timing based ranging technique. Multiple delayed, attenuated and phase rotated replicas of the originally transmitted signal of limited bandwidth are superimposed at the receiver antenna. In this section we have a closer look at the multipath error envelope (MEE).

The MEE, also known as multipath-profile, is a common way of illustrating multipath performance for a given bandwidth limited signal applied for time based ranging [9]. It shows the influence of a single multipath component with a dedicated magnitude, phase and delay relative to the LoS path, on the range estimation bias. Hence, the channel defined in (4) simplifies to

$$
h[n, \tau]=\beta_{1} \delta\left[n-\frac{\tau_{1}}{T_{\mathrm{s}}}\right]+\beta_{2} \delta\left[n-\frac{\tau_{2}}{T_{\mathrm{s}}}\right],
$$

with $\tau_{1}$ as the delay of the LoS path and $\tau_{2}$ as delay of the first multipath component. For the MEE we are only interested in relative delays, amplitude levels, and phases. The delay of the interfering multipath component is swept from $\tau_{2}>\tau_{1}$ up to a predefined limit of interest. The magnitude $\left|\beta_{2}\right|^{2}$ is set to a fixed level of, e.g., $3 \mathrm{~dB}$ lower than the magnitude of the LoS path, and the relative phase is swept between $0^{\circ}$ and $360^{\circ}$.

In this work, the MEE is evaluated as follows: we apply the incoherent ML estimator defined in (6) with the channel from (8), and set the noise term $z \equiv 0$ as we are only interested in the estimation bias. The power of the multipath component is $3 \mathrm{~dB}$ lower than the LoS path, and range estimation is based on the two-step approach: acquisition by maximum correlation peak detection and fine-synchronization in frequency-domain. Relative phases are swept within $\left[0^{\circ}, 360^{\circ}\right)$ in $5^{\circ}$ steps, and we average the obtained range estimation bias over these relative phases. This averaging is equivalent to a spatial average over the carrier phase. According to 3GPP-LTE we use a sampling frequency of $30.72 \mathrm{MHz}$ and an OFDM symbol length of $N=$ 2048. In contrast to 3GPP-LTE we allocate approximately $90 \%$ of the sampling bandwidth with $N_{\mathrm{u}}=1800$, which results in an effective bandwidth of $27 \mathrm{MHz}$ and an effective spatial sample length of $11.1 \mathrm{~m}$ with a propagation speed of $3 \times 10^{8} \mathrm{~m} \mathrm{~s}^{-1}$. The state of the art rectangular waveform always serves as reference. 


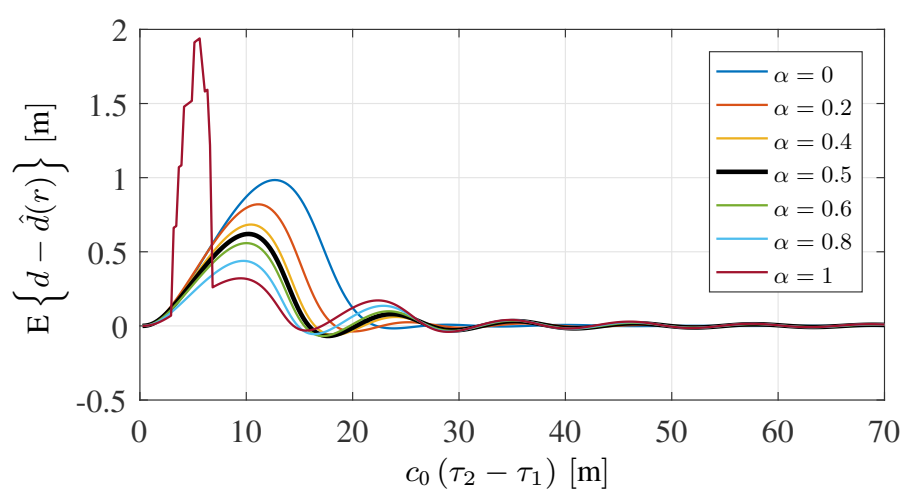

Fig. 5. MEE for the triangle waveform with selected waveform parameters $\alpha$ and averaged over all relative phases. The state of the art rectangular waveform is obtained with $\alpha=0.5$.

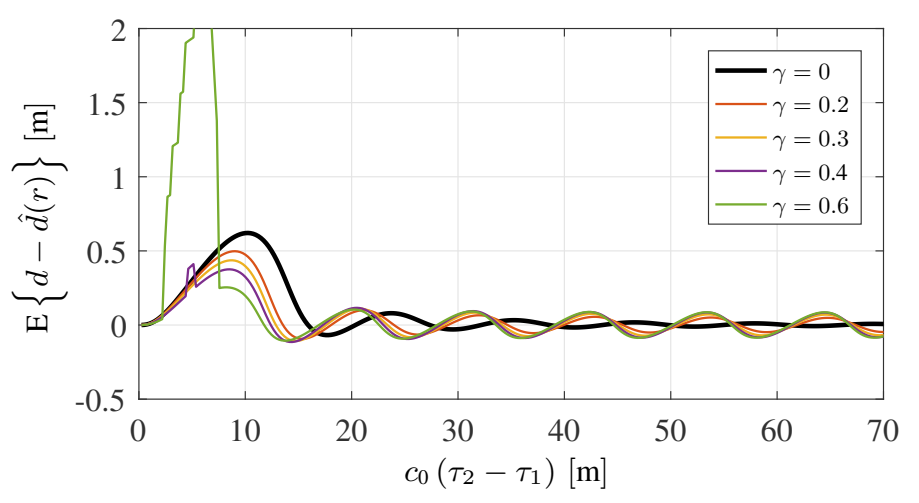

Fig. 6. MEE for the Dirac-rectangular waveform with selected waveform parameters $\gamma$ and averaged over all relative phases. The state of the art rectangular waveform is obtained with $\gamma=0$.

Fig. 5 shows the multipath error envelope averaged over all relative phases for the triangle waveform. Only some waveform parameters $\alpha$ are selected for illustration. We can clearly see the trade-off between the wider main correlation peak and reduced side lobes: for $\alpha=0$ the ranging bias vanishes for relative delays equivalent to $25 \mathrm{~m}$ and above, but the maximum ranging bias is higher compared to the rectangle waveform with $\alpha=0.5$. A narrower main correlation peak, e.g., for $\alpha=0.8$ results in a lower maximum ranging bias but results in a larger ranging bias at higher relative delays. Too high correlation side-lobes as for $\alpha=1$ result in unfavorable superpositions of the main correlation peak and the correlation peak of the multipath component. Maximum correlation peak detection in the acquisition step fails and estimation outliers occur. We can observe this from Fig. 5 with $\alpha=1$, where the ranging bias suddenly increases for relative delays around $5 \mathrm{~m}$. Hence, this waveform parameter should not be chosen for such a channel.

The proposed Dirac-rectangular waveform shows a similar result compared to the triangle waveform, see Fig. 6. We do not show results for parameter values $\gamma>0.6$, because beyond this $\gamma$ we always experience incorrect correlation peak detections and the estimation bias is off the chart. Compared to lower bound results in [6], [7] we obtain a much lower

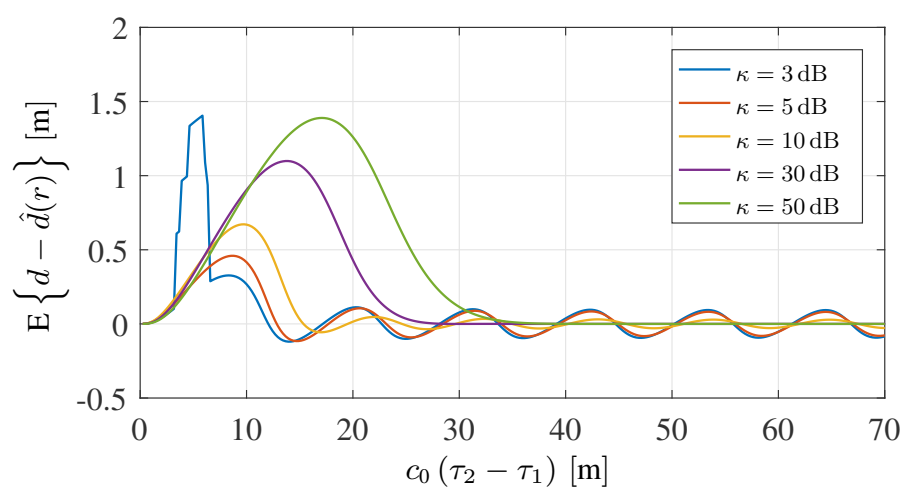

Fig. 7. MEE for the Dolph-Chebyshev waveform with selected waveform parameters $\kappa$ and averaged over all relative phases.

waveform parameter range for which this Dirac-rectangular waveform shows an improvement.

Fig. 7 shows the result for the Dolph-Chebyshev waveform and a selected number of side-lobe attenuation values $\kappa$. These results are comparable to the Dirac-rectangular waveform for small values of $\kappa$ because the PSDs are similar, see Fig. 2(a) and Fig. 3(a) for comparison.

Finally, we select the best MEE result from all three waveforms for which no detection outliers occur, and shortly discuss the result. Fig. 8 shows these three results together with the result from the rectangular waveform as reference. Compared to the state of the art rectangular waveform we can reduce the maximum average ranging estimation bias by about $42 \%$. We also observe that the difference between the triangle and the Dirac-rectangular waveform is negligible with respect to the maximum estimation bias. However, the estimation bias from the triangle waveform decays much faster over higher relative delays compared to the Dirac-rectangular waveform.

Naturally, one might ask in which channel scenarios our proposed waveforms and their respective parameters are beneficial based on the MEE evaluations. Let us consider two channel scenarios: an indoor channel with a very small delay spread and and outdoor channel with a larger delay spread and multipath clustering. In an indoor scenario the delay spread is commonly very small. Hence, the relative delay between the LoS path and any other multipath component is small. In such a scenario, we require a small range estimation bias at small relative delays but we don't need to consider higher relative delays. As a consequence, larger estimation biases resulting from higher correlation side-lobes at larger relative delays would not degrade an application's positioning performance, as larger relative delays simply do not occur. The Dirac-rectangular waveform would be a beneficial choice. For an outdoor channel scenario this relation is exactly opposite compared to the indoor channel scenario. For example, if clusters of multipath components with large relative delays occur, we might choose a Dolph-Chebyshev waveform with very small correlation side-lobes to suppress the interference resulting from multipath. 


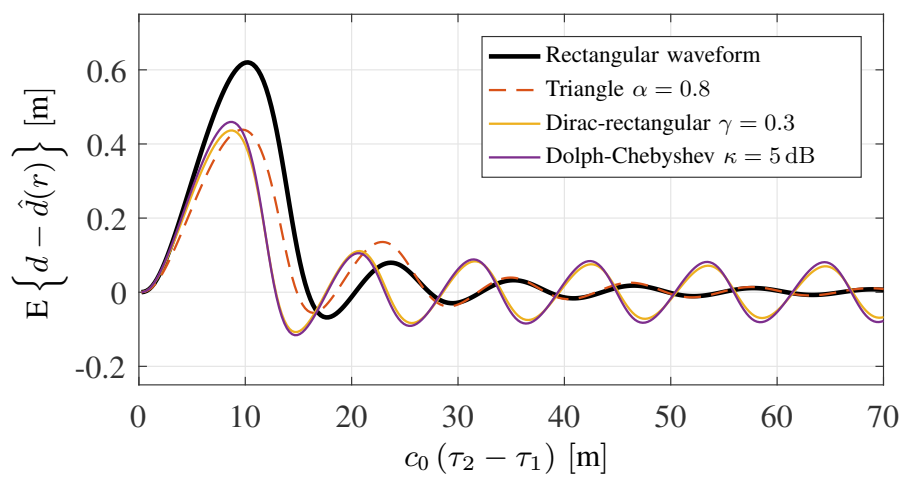

Fig. 8. MEE of all three waveforms with their respective best parameter Compared to the state of the art rectangular waveform we achieve an improvement of about $42 \%$.

TABLE I

OVERVIEW OF SELECTED WINNER-II SCENARIO PARAMETERS [10].

\begin{tabular}{l|c|c}
\multicolumn{1}{c|}{ WINNER-II scenario } & RMS delay spread & K-factor \\
\hline $\begin{array}{l}\text { A1, in building } \\
\text { Indoor office/residential }\end{array}$ & $38 \mathrm{~ns}$ & $7 \mathrm{~dB}$ \\
\hline $\begin{array}{l}\text { B1, hotspot } \\
\text { Typical urban micro-cell }\end{array}$ & $36 \mathrm{~ns}$ & $9 \mathrm{~dB}$ \\
\hline $\begin{array}{l}\text { B3, hotspot } \\
\text { Large indoor hall }\end{array}$ & $29 \mathrm{~ns}$ & $2 \mathrm{~dB}$ \\
\hline $\begin{array}{l}\text { C1, metropol } \\
\text { Suburban }\end{array}$ & $58 \mathrm{~ns}$ & $9 \mathrm{~dB}$ \\
\hline $\begin{array}{l}\text { C2, metropol } \\
\text { Typical urban macro-cell }\end{array}$ & $40 \mathrm{~ns}$ & $7 \mathrm{~dB}$ \\
\hline $\begin{array}{l}\text { D1, rural } \\
\text { Rural macro-cell }\end{array}$ & $15 \mathrm{~ns}$ & $7 \mathrm{~dB}$ \\
\hline $\begin{array}{l}\text { D2a, moving networks } \\
\text { BS-MRS, rural }\end{array}$ & $39 \mathrm{~ns}$ & $7 \mathrm{~dB}$
\end{tabular}

\section{WINNER-II ChanNel Model Evaluation}

As a next step we investigate the ranging performance of the proposed waveforms by using a state of the art channel model widely used in the communication community. The WINNER-II channel model is used and we selecte the model scenarios A1, B1, B3, C1, C2, D1, and D2a in the LoS case for numerical evaluation [10]. These numerical evaluations should have shown which specific waveform and waveform parameter should be chosen for a particular channel model scenario, such as indoor office, urban micro-cell, indoor hall, urban macrocell, and so on. Unfortunately, our numerical evaluations were inconclusive: we did not find significant correlations between the model scenarios and its model parameters and the optimally chosen waveform and waveform parameter. We investigated this issue further and identified the following circumstances which have lead to these inconclusive results: for all LoS scenarios in the WINNER-II channel model the RMS delay spread is approximately equal and the Rician Kfactor is approximately equal as well. Tab. I summarizes the RMS delay spread and Rician K-factor for the afore listed model scenarios. Hence, we cannot correlate our findings with the RMS delay spread as the RMS delay spread does not cover a wider range of values.

\section{Delay Spread Dependent Ranging ACCuracy}

The inconclusive results from WINNER-II channel evaluations in the previous section shows that we need a different channel model for our evaluations. At the end of our evaluations we want to give a clear answer in which environments particular waveforms and parameters should be chosen. We focus on LoS propagation conditions for ranging, and hence, the channel can properly be described in general by the Ricean $\mathrm{K}$-factor, and the RMS delay spread denoted as $\tau_{\mathrm{RMS}}$. The RMS delay spread in particular describes various outdoor and indoor environments very well: indoors $\tau_{\mathrm{RMS}}$ is in the order of about $100 \mathrm{~ns}$, and outdoors $\tau_{\mathrm{RMS}}$ can reach up to several $\mu \mathrm{s}$ depending on the carrier frequency.

\section{A. Channel Generation}

Several methods to generate channel realizations based on a given K-factor and $\tau_{\text {RMS }}$ are available in existing literature [11]. At this point we need to make clear that channel realizations with multipath components lying in fixed equidistant taps must not be chosen. In [11] this is described as method of equal distances. Such generated channels will lead to incorrect evaluation results as waveforms with null-values in the autocorrelation function at particular taps are easily found but in reality, multipath components seldom lie exactly on equidistant taps. Consequently, we chose the multipath delay generation method as described by WINNER-II in [10, Sec. 4.2] and adapted it to our needs.

Channel generation parameters are as follows: the Rician $\mathrm{K}$-factor is fixed to $7 \mathrm{~dB}$ as in [10, Tab. 4-5], we use an exponentially decaying power delay profile [10, Tab. 4-5], a total number of 20 rays for the multipath generation, and a varying RMS delay spread $\tau_{\text {RMS }}$ between $1 \mathrm{~ns}$ and $10 \mu \mathrm{s}$. Phases of all multipath rays and the LoS path are randomly generated and uncorrelated for each channel realization with uniform distribution. Additionally, the delay of the LoS path is known but uniformly distributed within $[0,1)$. The channel is interpolated and applied in frequency-domain, as we use OFDM modulated signals. $1 \times 10^{4}$ channel realizations are generated for evaluations.

OFDM signal parameters are as follows: the subcarrier spacing is $f_{\mathrm{sc}}=15 \mathrm{kHz}$ and we use three different $3 \mathrm{GPP}$ LTE bandwidths $100 \mathrm{MHz}, 20 \mathrm{MHz}$ and $5 \mathrm{MHz}$ [2]. The corresponding OFDM symbol lengths without cyclic prefix $N$ are therefore 8192, 2048 and 512 and the amount of allocable subcarriers $N_{\mathrm{u}}$ are 6666, 1200 and 300 respectively. Our chosen RMS delay spread value range requires an increased cyclic prefix length of $C=N / 2$ to consume the channel impulse response and to avoid inter-symbol interference (ISI).

\section{B. Performance Metric}

In many cases the ranging root mean square error (RMSE) is the chosen performance metric for snapshot based range estimation. A first evaluation however showed, that the achievable gain in RMSE for different optimally chosen waveforms over the RMS delay spread compared to the rectangular waveform is negligible. As mentioned in Sec. II the chosen waveforms 


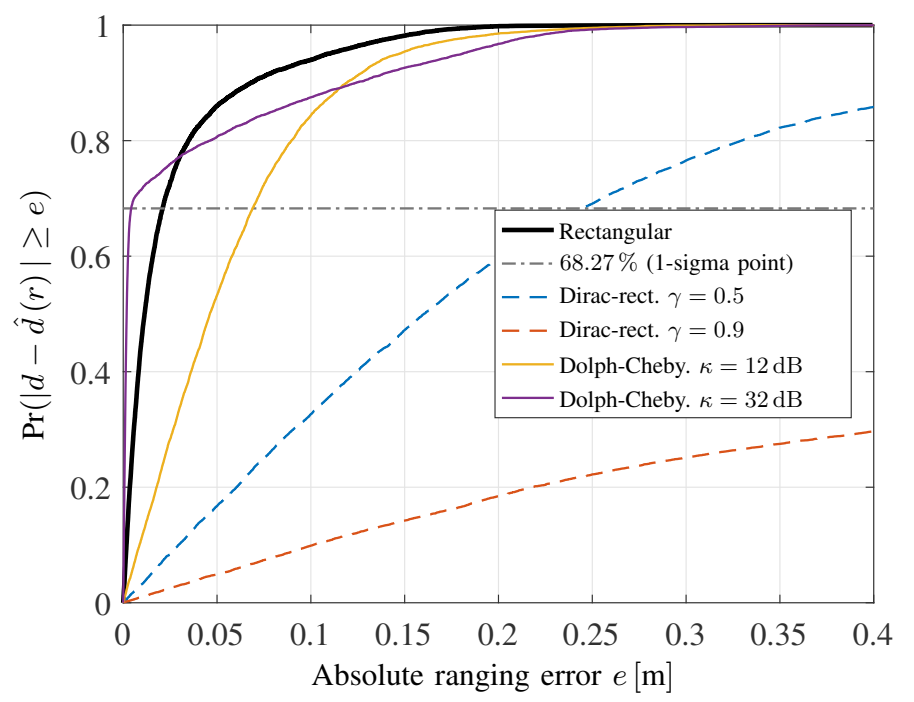

Fig. 9. CDF of the absolute ranging error for selected waveforms to illustrate the 1 -sigma cutting point. The bandwidth is $100 \mathrm{MHz}$ and $\tau_{\mathrm{RMS}}$ is $4 \mu \mathrm{s}$.

are simply windowing functions. Hence, for particular channel realizations we gain, for some we loose ranging performance, and in the average we do not gain at all. However, localization algorithms commonly employ tracking and the snapshot based ranging RMSE is not a well suited performance metric. The error distribution becomes more important if localization algorithms within a Bayesian framework are chosen.

In this work, we therefore focus on the 1-sigma cumulative distribution function (CDF) point of the absolute ranging error. Fig. 9 shows CDF curves of the absolute ranging error of selected waveforms for explanation. The rectangular waveform is always our reference. As we can see in this example, the Dolph-Chebyshev waveform with $\kappa=32 \mathrm{~dB}$ performs better compared to the rectangular waveform up to about $75 \%$ of the cases. We are thus interested in waveforms which result in a lower absolute ranging error $e$ in $68.27 \%$ of the cases compared to the rectangular waveform.

\section{Results}

At first we have a look at the absolute ranging error for the state of the art rectangular waveform over the RMS delay spread $\tau_{\text {RMS }}$ for SNR values of $-10 \mathrm{~dB}, 0 \mathrm{~dB}$ and $10 \mathrm{~dB}$ and the three bandwidths of $100 \mathrm{MHz}, 20 \mathrm{MHz}$ and $5 \mathrm{MHz}$. The SNR denotes the signal-to-noise ratio at the receiver input before the correlator/matched filter, and hence, does not include the correlation gain. Fig. 10 shows these results, which serve as reference and benchmark for the proposed waveforms in this work. For comparison we calculated the CRLB for the multipath-free case for the rectangular waveform. Fig. 10(a) shows the result for a bandwidth of $100 \mathrm{MHz}$. We observe a small ranging error for very small delay spreads, because the relative delay of interfering multipath components is so small that the channel becomes frequency-flat again. The ranging error increases for larger delay spreads and reaches a maximum at $\tau_{\mathrm{RMS}} \approx 30 \mathrm{~ns}$. This delay spread is typical

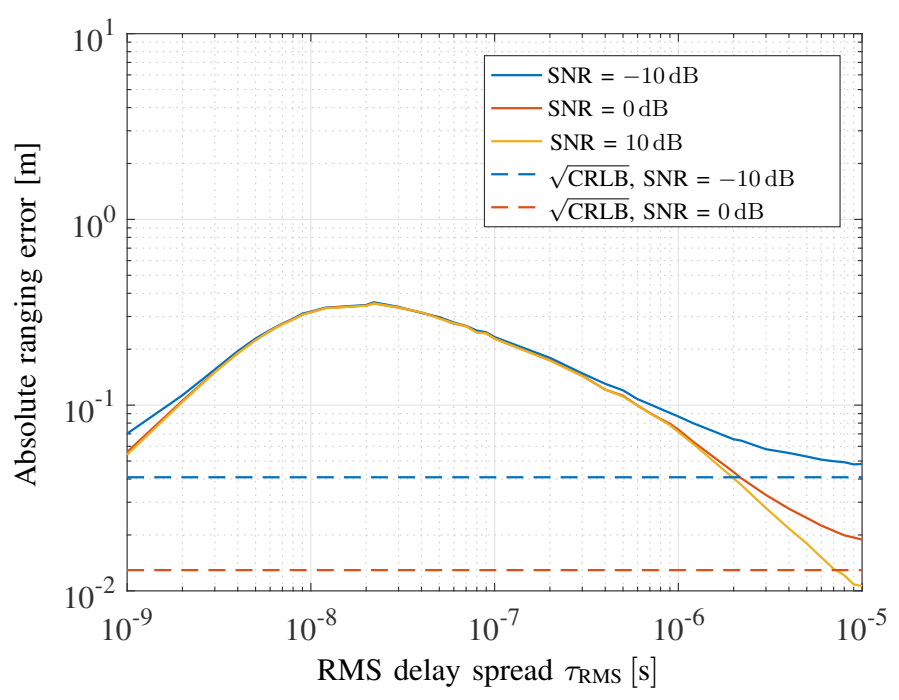

(a) Rectangular waveform with a bandwidth of $100 \mathrm{MHz}$.

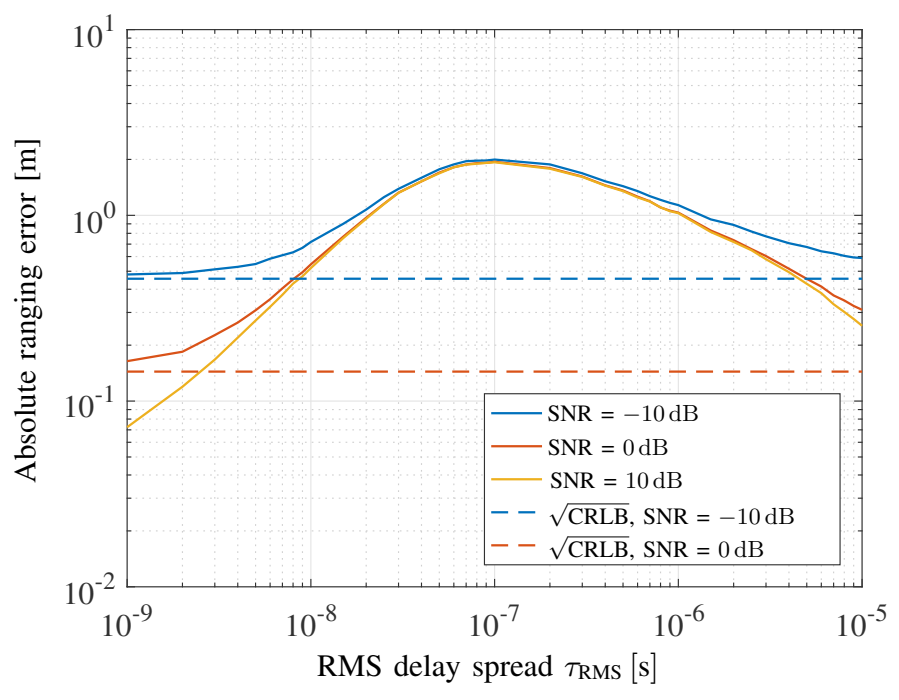

(b) Rectangular waveform with a bandwidth of $20 \mathrm{MHz}$.

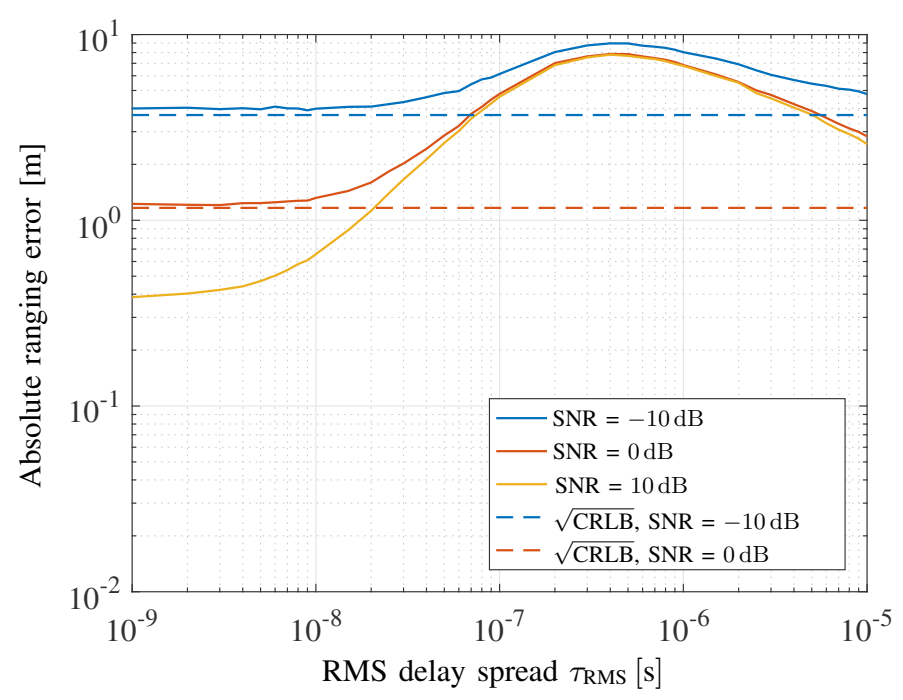

(c) Rectangular waveform with a bandwidth of $5 \mathrm{MHz}$.

Fig. 10. Absolute ranging error based on the 1-sigma CDF cutting point $(68.27 \%)$ of the rectangular waveform for reference. The square-root of the CRLB for multipath-free channels is additionally shown for SNRs of $-10 \mathrm{~dB}$ and $0 \mathrm{~dB}$. 
for indoor scenarios, see Tab. I. For larger delay spreads reaching $10 \mu \mathrm{s}$ we observe a smaller ranging error because the probability of interfering multipath components with small relative delay is lower due to the exponentially decaying power delay profile. Additionally, interfering autocorrelation side-lobes from interfering multipath components with larger relative delay are smaller, as the side-lobes decay fast for the rectangular waveform. Two interesting aspects are visible: the receiver SNR does not play a role for $\tau_{\text {RMS }}$ between $2 \mathrm{~ns}$ and $700 \mathrm{~ns}$, and the ranging error converges to specific values. The first aspect highlights that the ranging error is solely dominated by the frequency-selective channel resulting in an increased ranging bias. Regardless of the SNR we cannot obtain a lower ranging error. The second aspect highlights that the ranging error becomes dominated by AWGN, and converges to the calculated square-root of the CRLB. A smooth transition between those two aspects is also visible. Once the system is noise-limited only, the results from [5], [6] are applicable. Results for bandwidths of $20 \mathrm{MHz}$ and $5 \mathrm{MHz}$ shown in Fig. 10(b) and Fig. 10(c) are similar. Particularly for $5 \mathrm{MHz}$ we clearly see the noise domination for delay spreads up to $30 \mathrm{~ns}$ for an SNR of $-10 \mathrm{~dB}$.

Secondly, we calculate the CDF of the absolute ranging error for all waveform and parameter combinations, and select the combination with the smallest absolute error at the 1sigma point. This selection is equivalent to a genie-algorithm knowing the K-factor and RMS delay spread of the channel a-priori and providing us the best parameter combination. We define a ranging error reduction factor

$$
G=\frac{e_{\mathrm{ref}}}{e_{\mathrm{opt}}},
$$

with $e_{\text {ref }}$ as reference error obtained from the state of the art rectangular waveform, and $e_{\mathrm{opt}}$ as error from the optimally chosen waveform parameter, see also Fig. 9. Hence, $G$ directly indicates how much better a proposed waveform is compared to state of the art.

Fig. 11 shows the error reduction factor for all three waveforms with optimally chosen waveform parameters at a signal bandwidth of $100 \mathrm{MHz}$. Let us focus on the triangle waveform in Fig. 11(a) first: $G$ is negligible for $\tau_{\text {RMS }}$ below $1 \mu$ s and does not show an SNR dependency. Above $1 \mu$ s we clearly see a significant improvement, particularly for higher SNRs. At this point we observe the ranging performance limitation based on the frequency-selective channel and AWGN again: the lower plot in Fig. 11(a) shows the corresponding optimal waveform parameter. $\alpha$ converges to 0 for high SNRs resulting in a PSD concentration around the DC-carrier, see Fig. 1(a), and faster decaying autocorrelation side-lobes. For lower SNRs we transition to the AWGN limitation. Hence, $\alpha$ converges to 1 resulting in a PSD concentration at the spectrum's edge, see SNR values of $-10 \mathrm{~dB}$ and $0 \mathrm{~dB}$. Minor variations of $\alpha$ for a particular SNR over $\tau_{\text {RMS }}$ result from the limited number of channel realizations and resulting minor variations in the calculated CDFs. The Dirac-rectangular waveform shows an improvement at RMS delay spreads around $10 \mathrm{~ns}$ to $100 \mathrm{~ns}$,
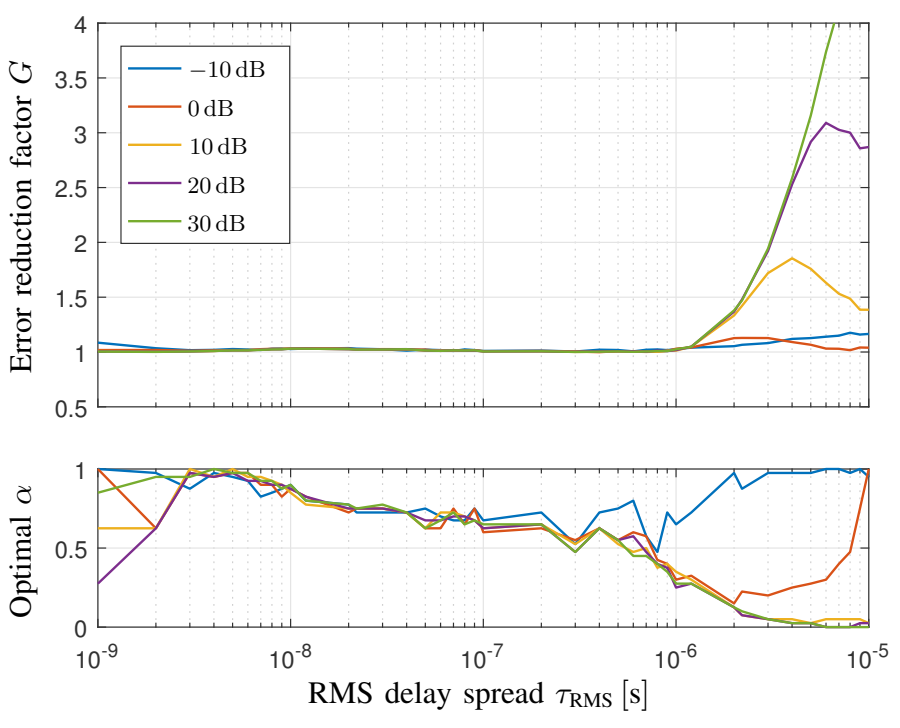

(a) Triangle waveform.
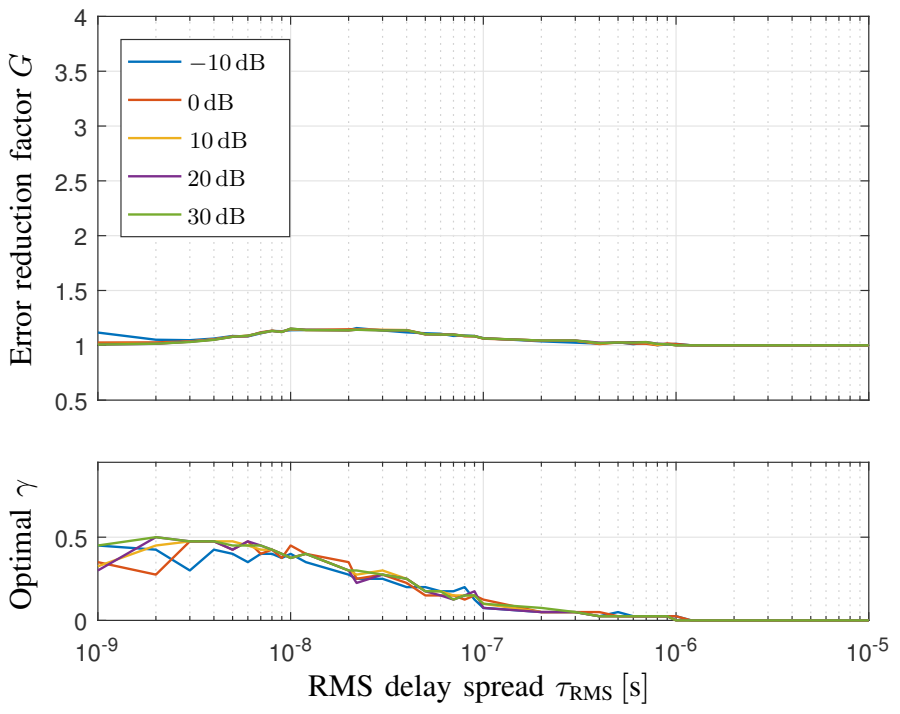

(b) Dirac-rectangular waveform.
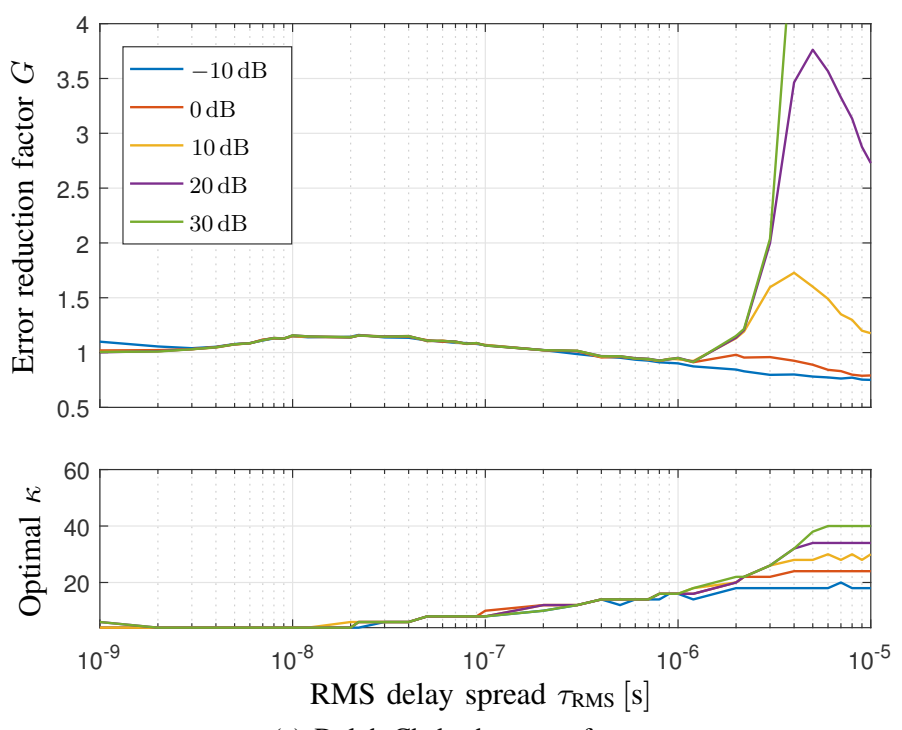

(c) Dolph-Chebyshev waveform.

Fig. 11. Resulting 1-sigma point ranging gain for a bandwidth of $100 \mathrm{MHz}$ and optimally chosen waveform parameters. Colors represent different SNRs. 

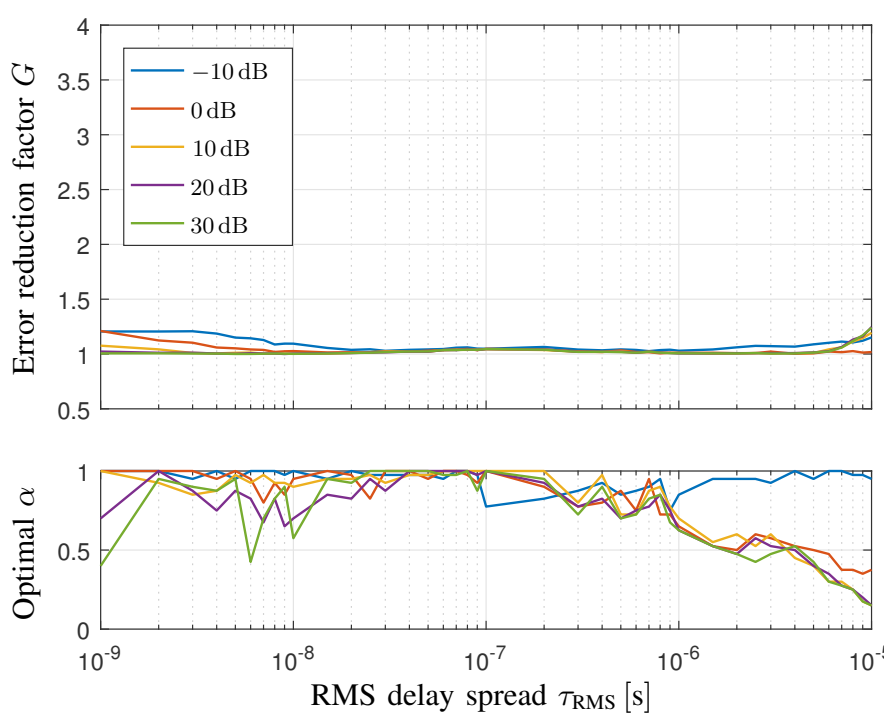

(a) Triangle waveform.
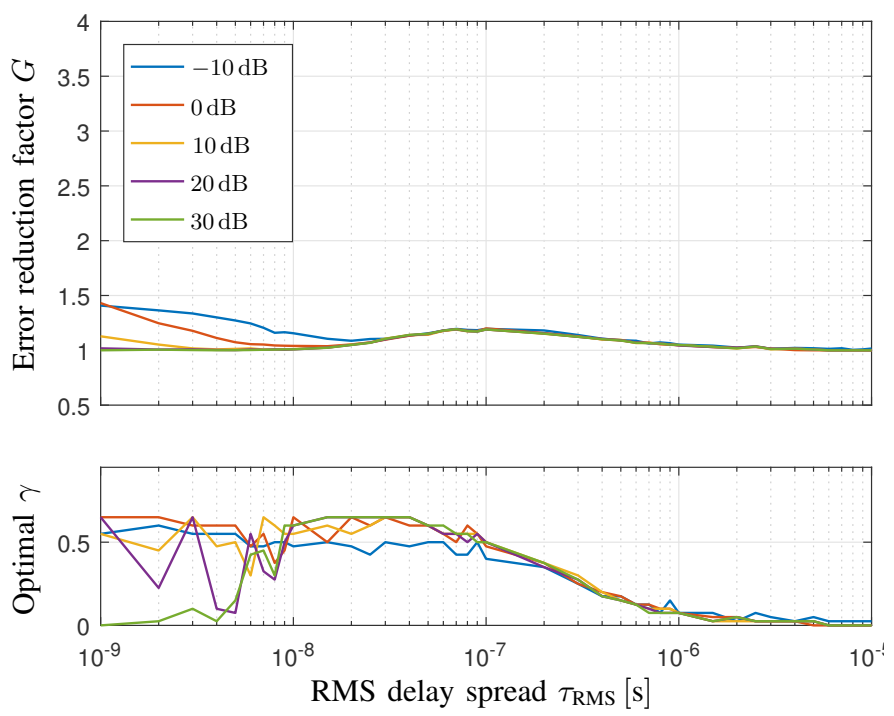

(b) Dirac-rectangular waveform.
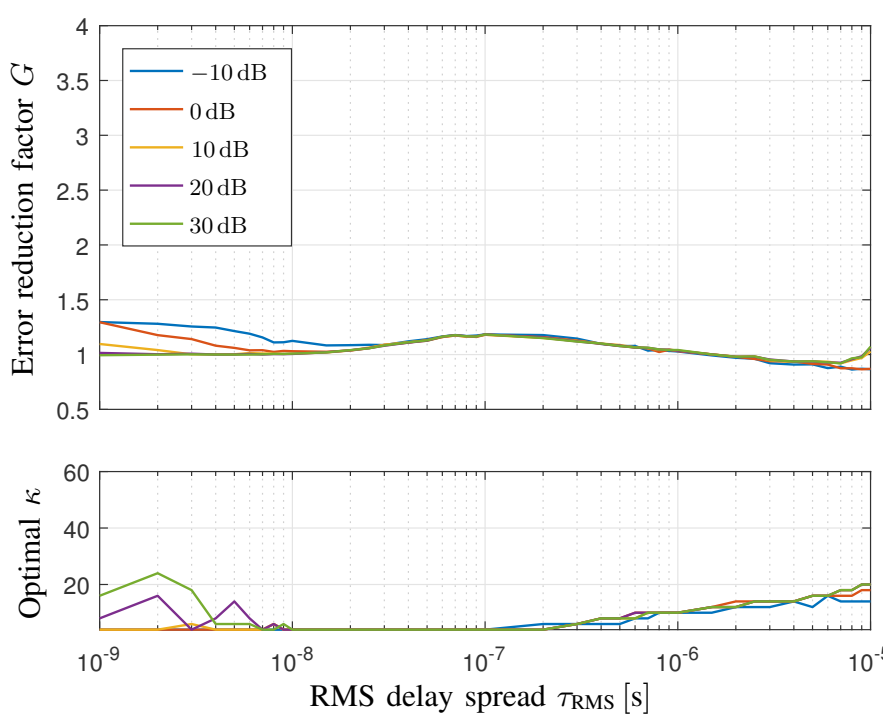

(c) Dolph-Chebyshev waveform.

Fig. 12. Resulting 1-sigma point ranging gain for a bandwidth of $20 \mathrm{MHz}$ and optimally chosen waveform parameters. Colors represent different SNRs.
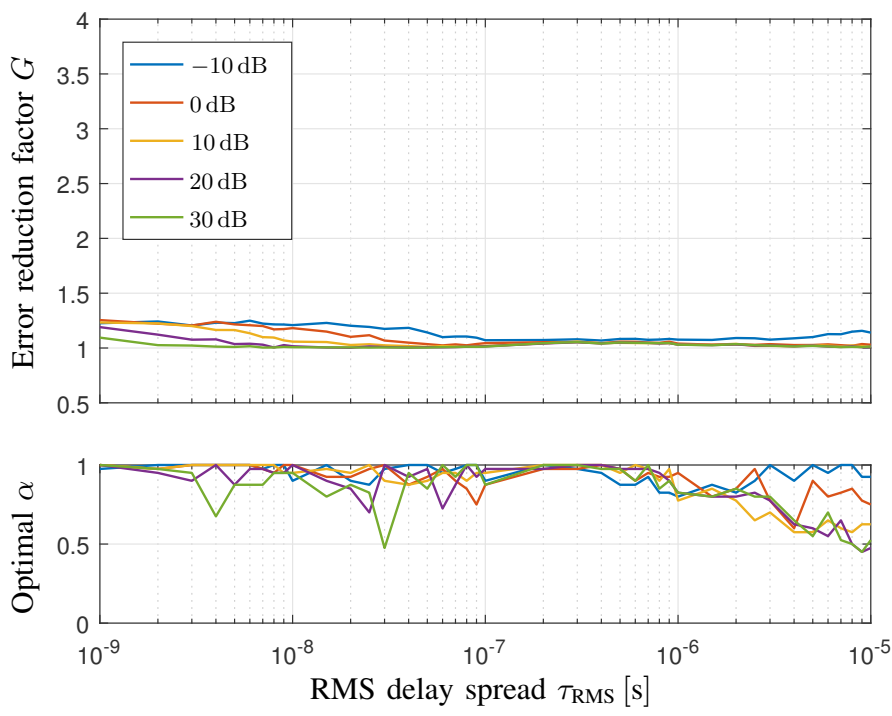

(a) Triangle waveform.
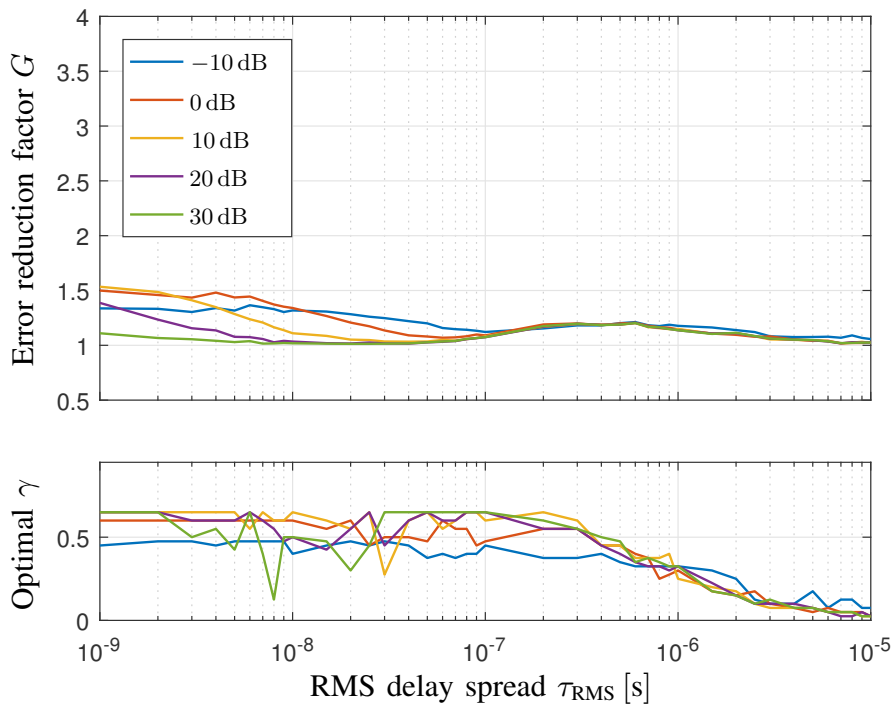

(b) Dirac-rectangular waveform.
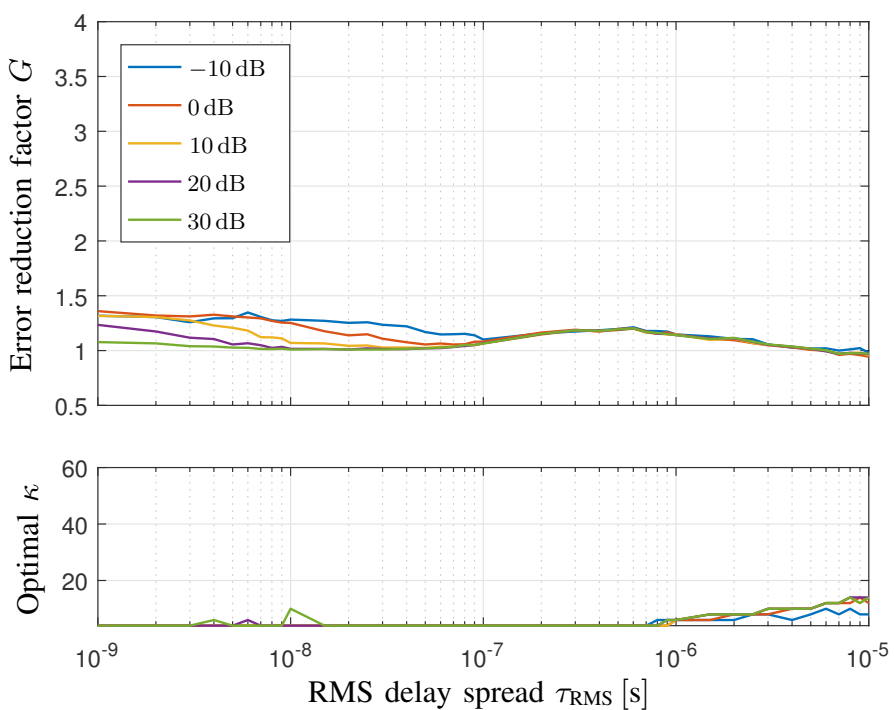

(c) Dolph-Chebyshev waveform.

Fig. 13. Resulting 1-sigma point ranging gain for a bandwidth of $5 \mathrm{MHz}$ and optimally chosen waveform parameters. Colors represent different SNRs. 
see Fig. 11(b). An improvement at larger $\tau_{\text {RMS }}$ is not possible because we can not concentrate the PSD around the DCcarrier. $\kappa$ converges to 0 which represents the rectangular waveform. We already observed in Sec. II and Fig. 3(a) that the Dolph-Chebyshev waveform is very similar to the Diracrectangular waveform for small side-lobe attenuation values $\kappa$. The ranging error reduction factor is similar compared to the Dirac-rectangular waveform for $\tau_{\text {RMS }}$ between $10 \mathrm{~ns}$ and 100 ns. Additionally, we also gain at larger RMS delay spreads for SNR values greater than $0 \mathrm{~dB}$. However, we loose at low SNRs compared to the rectangular waveform. The maximum achievable $G$ for $\tau_{\text {RMS }}$ between $10 \mathrm{~ns}$ and $100 \mathrm{~ns}$ is 1.161 .

Results for signal bandwidths of $20 \mathrm{MHz}$ and $5 \mathrm{MHz}$ depicted in Fig. 12 and Fig. 13 are similar compared to previously described results for $100 \mathrm{MHz}$. Keeping in mind the reference in Fig. 10, we observe much larger RMS delay spread value ranges where the ranging error is limited by AWGN and not by the frequency-selective channel itself. As a result we can see that for $\tau_{\text {RMS }}$ below $10 \mathrm{~ns}$ for $20 \mathrm{MHz}$ and below $100 \mathrm{~ns}$ for $5 \mathrm{MHz}$ : optimal waveform parameters across all waveform types converge to values with a PSD concentration at the spectrum's edge. Ranging error reduction factors of about 1.5 can be obtained in the AWGN limitation case and about 1.2 in the frequency-selective channel limitation case. Based on these results with lower signal bandwidths we can also state that the Dirac-rectangular waveform is a more optimal waveform compared to the state of the art rectangular waveform.

\section{CONCLUSION AND OUTLOOK}

Based on our numerical evaluations with varying RMS delay spread we can conclude as follows: the ranging error can be reduced by a factor between 1.2 and over 5 for optimally chosen waveforms and parameters compared to a state of the art rectangular PSD. It is important to say that we used a single-channel-tap correlation receiver also known as DLL in GNSS receivers for range estimation. Such an estimator can easily be implemented in existing communication receivers. Our results show which waveform and parameter should be chosen, given a particular channel RMS delay spread and signal bandwidth.

Further investigations should focus on deriving a theoretical framework to easily assess the resulting ranging performance over time-dispersive channels, as our conducted numerical evaluations are very time-consuming. With such a theoretical framework, additional parameters sweeps should be executed, e.g., more fine-grained systems bandwidths and different Ricean K-factors. The sensitivity of waveform parameter mismatch on ranging performance should also be part of further investigations.

\section{ACKNOWLEDGMENT}

This work has been performed in the framework of the German Aerospace Center (DLR) project Dependable Navigation, and has been partially supported by the EU project HIGHTS (High precision positioning for cooperative ITS applications) MG-3.5a-2014-636537. We would also like to thank our colleagues Thomas Jost and Siwei Zhang for further discussions and given hints with respect to the WINNER-II channel model.

\section{REFERENCES}

[1] H. Wymeersch, J. Lien, and M. Win, "Cooperative Localization in Wireless Networks," Proc. IEEE, vol. 97, no. 2, pp. 427 - 450, Feb. 2009.

[2] G. O. Partners, "LTE; Evolved Universal Terrestrial Radio Access (EUTRA); Physical channels and modulation (3GPP TS 36.211 version 8.7.0 Release 8)," European Telecommunications Standards Institute (ETSI), Tech. Rep., 2009.

[3] J. G. Andrews, S. Buzzi, W. Choi, S. V. Hanly, A. Lozano, A. C. K. Soong, and J. C. Zhang, "What will 5g be?" IEEE J. Sel. Areas Commun., vol. 32, no. 6, pp. 1065 - 1082, Jun. 2014.

[4] P. Banelli, S. Buzzi, G. Colavolpe, A. Modenini, F. Rusek, and A. Ugolini, "Modulation formats and waveforms for $5 \mathrm{~g}$ networks: Who will be the heir of ofdm?: An overview of alternative modulation schemes for improved spectral efficiency," IEEE Signal Process. Mag., vol. 31, no. 6, pp. 80 - 93, Nov. 2014.

[5] A. Dammann, R. Raulefs, and S. Zhang, "On prospects of positioning in 5G," in Proceedings IEEE ICC 2015 - Workshop on 5G \& Beyond Enabling Technologies and Applications, London, UK, Jun. 2015.

[6] R. Raulefs, A. Dammann, T. Jost, M. Walter, and S. Zhang, "The 5G localization waveform," in Proc. ETSI Workshop on Future Radio Technologies focusing on Air Interfaces, Sophia Antipolis, France, Jan. 2016.

[7] A. Dammann, T. Jost, R. Raulefs, M. Walter, and S. Zhang, "Optimizing waveforms for positioning in 5G," in IEEE 17th International Workshop on Signal Processing Advances in Wireless Communications (SPAWC 2016), Edinburgh, United Kingdom, Jul. 2016.

[8] E. Staudinger and C. Gentner, "TDoA subsample delay estimator with multiple access interference mitigation and carrier frequency offset compensation for OFDM based systems," in Proceedings of $8^{\text {th }}$ Workshop on Positioning Navigation and Communication, Dresden, Germany, Apr. 2011 , pp. $33-38$

[9] M. Irsigler, "Multipath Propagation, Mitigation and Monitoring in the Light of Galileo and the Modernized GPS," Ph.D. dissertation, Universität der Bundeswehr München, Jul. 2008.

[10] "WINNER II Deliverable D1.1.2: WINNER II Channel Models," Sep. 2007. [Online]. Available: http://www.ist-winner.org/deliverables.html

[11] M. Pätzold, A. Szczepanski, and N. Youssef, "Methods for modeling of specified and measured multipath power-delay profiles," IEEE Trans. Veh. Technol., vol. 51, no. 5, pp. 978 - 988, Sep. 2002. 